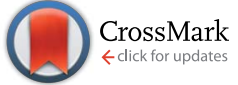

Cite this: RSC Adv., 2017, 7, 4891

Received 5th December 2016

Accepted 4th January 2017

DOI: $10.1039 / \mathrm{c} 6 \mathrm{ra} 27760 \mathrm{~h}$

www.rsc.org/advances

\section{High-yield and rapid synthesis of ultrathin silver nanowires for low-haze transparent conductors $\dagger$}

\author{
Yang Liu, Yingying Chen, Rui Shi, Lijun Cao, Zhan Wang, Tao Sun, Jianjian Lin, \\ Juqing Liu* and Wei Huang*
}

Ultrathin silver nanowires (Ag NWs) have been rapidly synthesized by using $\mathrm{NaBr}$ and $\mathrm{AgCl}$ as conucleant, with a high yield of $90 \%$. The average diameter and length of $\mathrm{Ag} \mathrm{NWs}$ is $30 \mathrm{~nm}$ and $11 \mu \mathrm{m}$, respectively. The resulting conductor exhibits high ratio of DC conductivity to optical conductivity of 162 , low sheet resistance of $12.2 \Omega \mathrm{sq}^{-1}$ at $83.3 \%$ and low haze of $0.96 \%$ at $95.7 \%$.
Transparent conducting electrodes (TCEs) are essential components in various optoelectronic devices such as solar cells, organic light emitting diodes, liquid crystal displays, touch screens, and smart windows. ${ }^{1,2}$ Nowadays, the most common conductive material used in TCEs is indium tin oxide (ITO). However, poor mechanical flexibility, limited supply, high cost, and the vacuum forming process of the ITO layer have become an obstacle for its further development in conductors, together with limitations in future electronics. ${ }^{3}$ Several alternative materials have been developed to replace ITO, such as carbon nanotubes (CNTs), ${ }^{4-6}$ conductive polymers, ${ }^{5,6}$ graphene, ${ }^{4-7}$ metal nanowires, ${ }^{4,5,7}$ and hybrid electrodes. ${ }^{5,7}$ Among them, metal nanowire conductive materials, mainly silver nanowires (Ag NWs) and copper nanowires ( $\mathrm{Cu} \mathrm{NWs),} \mathrm{have} \mathrm{been} \mathrm{widely} \mathrm{explored} \mathrm{for} \mathrm{their} \mathrm{promising}$ opto-electrical properties and outstanding mechanical flexibility. It is noted that Ag NWs exhibit excellent electrical conductivity, superior electrical, and thermal conductivities compared with other metals. ${ }^{1-3,8-15}$ Therefore, Ag NWs are widely considered to be the optimal material for fabricating flexible electronics.

To synthesize Ag NWs, numerous methods have been employed, such as template wetting and polyol. ${ }^{16,17}$ The polyol method has been recognized as one of the most promising processes to reliably prepare $\mathrm{Ag}$ NWs, with the advantage of facile and controllable synthesis, and large-scale manufacturing and low-cost potential, ${ }^{11,13-15}$ to obtain the high performance Ag NW conductors, tremendous efforts have been made to synthesis uniform $\mathrm{Ag}$ NWs with long length and thin diameter. ${ }^{10-15,18-24}$ For example, Bergin et al. ${ }^{26}$ reported the synthesis of Ag NWs by the polyol method, with a thin diameter of $42 \mathrm{~nm}$. However, the nanowires were very short, with a length of $3 \mu \mathrm{m}$. More recently, Kim et al. ${ }^{12}$ successfully demonstrated the

Key Laboratory of Flexible Electronics (KLOFE), Institute of Advanced Materials (IAM), Jiangsu National Synergistic Innovation Center for Advanced Materials (SICAM), Nanjing Tech University (NanjingTech), 30 South Puzhu Road, Nanjing 211816, P. R. China.E-mail: iamjqliu@njtech.edu.cn; iamwhuang@njtech.edu.cn

$\dagger$ Electronic supplementary information (ESI) available. See DOI: $10.1039 / \mathrm{c} 6 \mathrm{ra} 27760 \mathrm{~h}$ synthesis of high quality Ag NWs with an average length of 13.5 $\mu \mathrm{m}$ and diameter up to $62.5 \mathrm{~nm}$. Thinner diameter $\mathrm{Ag} \mathrm{NW}$ formation can be achieved when high pressure is applied in an autoclave reactor, instead of the typical synthesis under atmospheric pressure. ${ }^{25}$ Zhang, et al. ${ }^{37}$ reported a facile one-pot polyol method to synthesis $\mathrm{Ag}$ NWs by using $\mathrm{KBr}$ as a conucleant to $\mathrm{NaCl}$ with long reaction time. The aspect ratio is crucial for the opto-electric properties of Ag NWs. Lee et al. ${ }^{18}$ shows short $(L=10.2 \mu \mathrm{m}) \mathrm{Ag}$ NW films $\left(T=77.21 \%, R_{\mathrm{s}}=34 \Omega\right.$ $\left.\mathrm{sq}^{-1}\right)$, long $(L=95.1 \mu \mathrm{m}) \mathrm{Ag}$ NW films $\left(T=96 \%, R_{\mathrm{s}}=186 \Omega\right.$ $\left.\mathrm{sq}^{-1}\right)$. Preston et al. ${ }^{13}$ shows large diameters $(D=150 \mathrm{~nm}) \mathrm{Ag}$ NW films $\left(T=75 \%, R_{\mathrm{S}}=135 \Omega \mathrm{sq}^{-1}\right)$, small diameters $(D=60$ $\mathrm{nm}) \mathrm{Ag}$ NW films $\left(T=78 \%, R_{\mathrm{s}}=45 \Omega \mathrm{sq}^{-1}\right)$. Therefore, high yield and rapid synthesis of ultrathin Ag NWs is necessary in commercial application of the emerging Ag NW conductors.

In this work, we report a modified polyol approach, using $\mathrm{NaBr}$ and $\mathrm{AgCl}$ as conucleant, for rapid synthesis of ultrathin, high quality, and monodispersed Ag NWs, with an average diameter of $30 \mathrm{~nm}$ and a mean length of $11 \mu \mathrm{m}$. Impressively, this efficiency approach is also associated with high yield of ultrathin Ag NW products, with a value up to $90 \%$. The resulting ultrathin Ag NW conductors show excellent conductivity, optical transparency, and low haze in comparison with previous reports. All these advances are expected to enable more possibilities for use of Ag NWs in future emerging conductors.

In order to synthesize an ultrathin $\mathrm{Ag}$ NWs suspension in ethanol, $0.012 \mathrm{~g} \mathrm{NaBr}$ (sodium bromide) was first added in $14 \mathrm{~mL}$ ethylene glycol (EG) containing $0.66 \mathrm{~g}$ polyvinylpyrrolidone (PVP, $M_{\mathrm{w}}=1300000$ ), and then the mixture was stirred at $200 \mathrm{rpm}$ and thermally stabilized at $165{ }^{\circ} \mathrm{C}$. Secondly, $0.05 \mathrm{~g}$ silver chloride $(\mathrm{AgCl})$ in a $2 \mathrm{~mL}$ EG solution was added to the mixture.

After three minutes, $4 \mathrm{~mL}$ of EG solution containing $0.22 \mathrm{~g}$ $\mathrm{AgNO}_{3}$ was injected with syringe using at a rate of $0.4 \mathrm{~mL} \mathrm{~min}{ }^{-1}$, the reaction was left for $30 \mathrm{~min}$ to allow the nanowires to grow. Finally, the Ag NW product was collected via centrifugation, washed with ethanol; the resulting suspension was used for subsequent characterization and conductor fabrication. 
Fig. 1 shows scanning electron microscopy (SEM) images and size distribution of the reaction product synthesized under different growth conditions. Reducing $\mathrm{AgNO}_{3}$ by using $\mathrm{AgCl}$ for the nucleation of silver seed resulting in synthesis of thin $\mathrm{Ag}$ NWs, and irregular silver nanoparticles (Ag NPs) were observed without addition of $\mathrm{AgCl}$ and $\mathrm{NaBr}$, meaning reducing solvents plays a key role in achieving nanowire features. Moreover, ultrathin $\mathrm{Ag}$ NWs were synthesized by using $\mathrm{NaBr}$ and $\mathrm{AgCl}$ as conucleant. The thin Ag NWs exhibit a diameter of $49.5 \pm$ $15.0 \mathrm{~nm}$ and length of $7.0 \mu \mathrm{m}$ (the size distribution of Ag NWs were counted by a nano measurer), which is comparable to that by gram-scale polyol synthesis ${ }^{26}$ but are of significantly higher quality than those of previous methods..$^{10,12,29}$ Compared to the thin Ag NWs, our ultrathin nanowires have a much smaller diameter and longer length, with the value of $30.4 \pm 9.0 \mathrm{~nm}$ and $11.0 \mu \mathrm{m}$, respectively. This diameter thinning from $50 \mathrm{~nm}$ to $30 \mathrm{~nm}$ after the introduction of $\mathrm{NaBr}$ during the reduction process, indicates the role of $\mathrm{NaBr}$ being crucial in synthesizing ultrathin Ag NWs.

To understand the growth mechanism and subsequent morphology, we propose that the diameter decrease in the present method could be attributed to the lower solubility product $\left(K_{\mathrm{sp}}\right)$ of $\mathrm{AgBr}$ compared to that of $\mathrm{AgCl}$. Tang et al. ${ }^{27}$ reported that Ag NPs were actually produced from $\mathrm{AgCl}$ by heating an $\mathrm{AgCl}$ solution, and the concentration of silver nuclei plays a crucial role for formation and the size of silver seeds. ${ }^{15,28}$ It is well known that $K_{\mathrm{sp}}$ of $\mathrm{AgCl}$ is higher than that of $\mathrm{AgBr}$, meaning that more Ag nuclei are generated during the reduction of silver ions by $\mathrm{AgCl}$, resulting in high concentration of $\mathrm{Ag}$ nuclei, which trigger the formation of large-size silver seeds. By injecting $\mathrm{AgNO}_{3}$ slowly, the amount of free $\mathrm{Ag}^{+}$ions in solution for $\mathrm{AgCl}$ system is higher than that for the $\mathrm{AgCl}$ and $\mathrm{NaBr}$ hybrid system, the kinetics of the reduction process of $\mathrm{AgNO}_{3}$ are slowed down due to the lower $K_{\mathrm{sp}}$ of $\mathrm{AgBr}$, resulting in a lower concentration of $\mathrm{Ag}^{+}$ions, which prefer to form ultrathin $\mathrm{Ag}$ NWs through small-size Ag seeds as initiation. Moreover, our method has a quicker reaction time overall than those previously reported $\mathrm{d}^{4,11,14,15}$ at $30 \mathrm{~min}$. Hence, this technique may be a very promising solution for the mass-production of low-cost Ag NWs.

Although well established in characterization technology, UV-visible spectroscopy has been used to investigate the morphological evolution of silver nanostructures due to the frequencies of surface plasmon resonances. ${ }^{30,31}$ Using this method, we obtained UV-visible absorption spectra of the final products under different growth conditions, as shown in Fig. 2

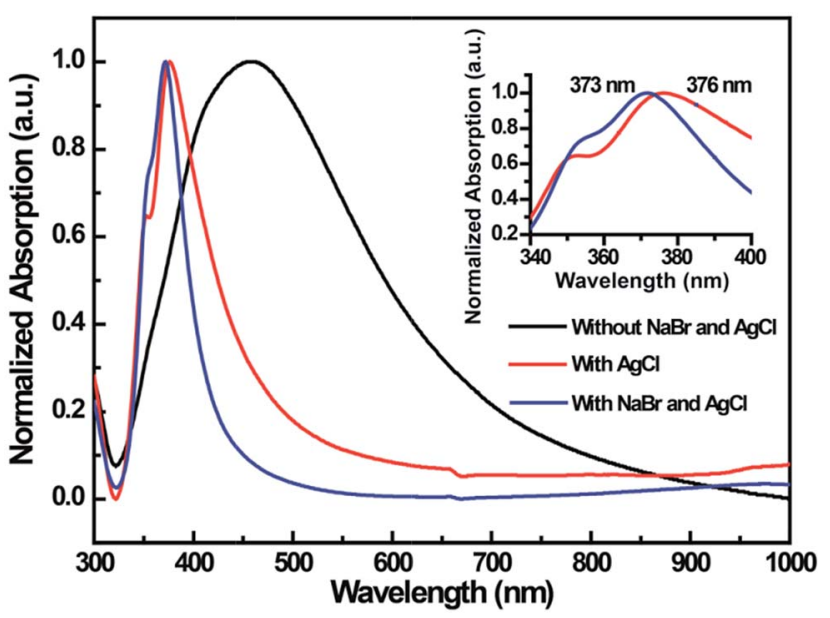

Fig. 2 UV-visible absorption spectra of the final products under different growth conditions. The inset represents the corresponding selected peaks ranging from $340 \mathrm{~nm}$ to $400 \mathrm{~nm}$.
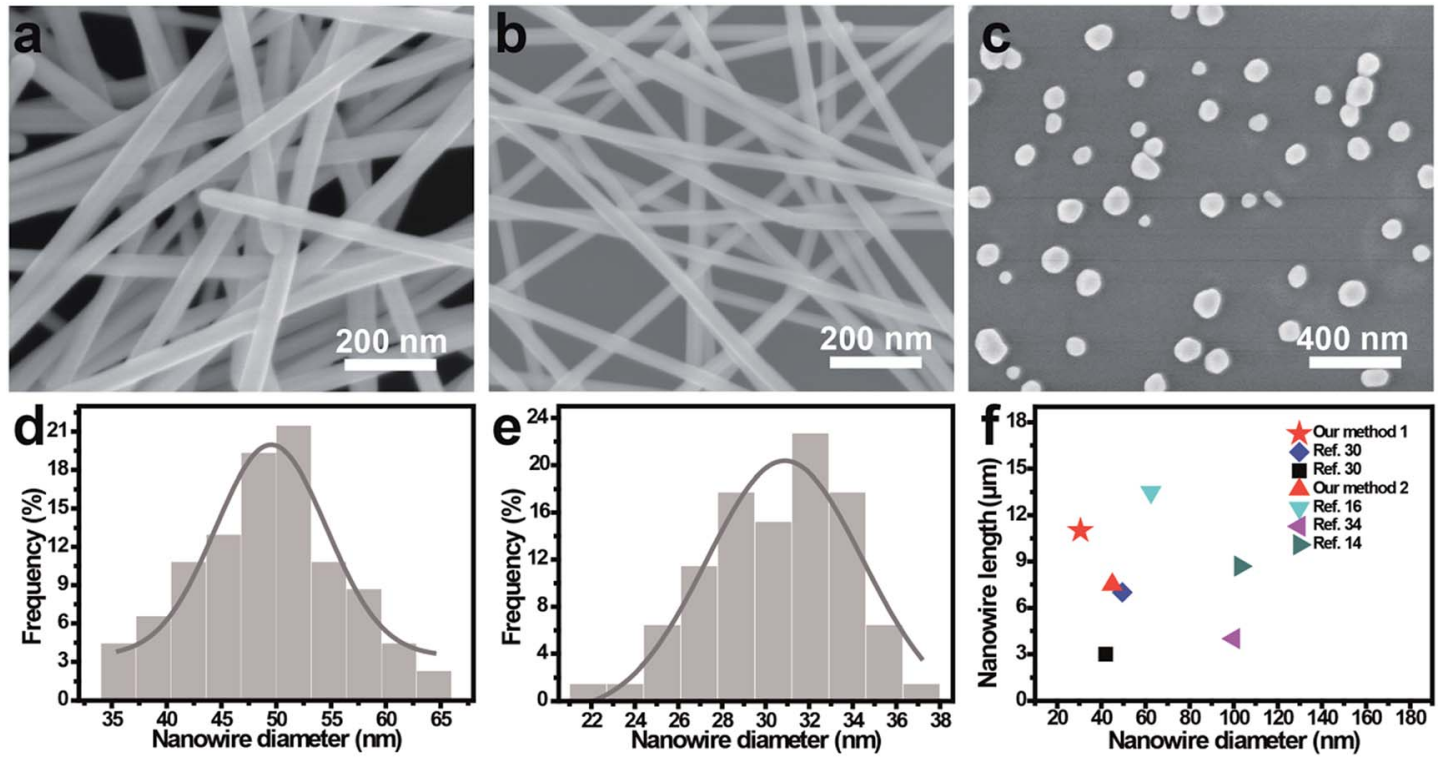

Fig. 1 SEM images of the products (a) with addition of $\mathrm{AgCl}$, (b) with addition of $\mathrm{AgCl}$ and $\mathrm{NaBr}$, (c) without addition of $\mathrm{AgCl}$ and $\mathrm{NaBr}$. Histogram show the diameter distribution of (d) thin Ag NWs and (e) ultrathin Ag NWs, which corresponding to product (a) and (b), respectively. (f) Diameter and length comparison of Ag NW products synthesized using polyol process method in the literature. 
typical broad peak at 453-463 nm was observed for the products without $\mathrm{AgCl}$ and $\mathrm{NaBr}$, indicating the formation of $\mathrm{Ag}$ NPs. However, a narrow peak at $376 \mathrm{~nm}$ with a distinct shoulder was found for thin $\mathrm{Ag}$ NWs, which is consistent with previous results. ${ }^{15,32}$ Impressively, the ultrathin Ag NWs exhibit a sharp peak at $373 \mathrm{~nm}$ with a slight shoulder, meaning the diameter decrease for Ag NWs is accompanied by a blue shift and narrow bandwidth of UV absorption spectra. In addition, the structure of ultrathin $\mathrm{Ag}$ NWs were studied by X-ray diffraction (XRD) shown in Fig. S1. $\dagger$ The nanowires exhibit four typical peaks, which corresponded to the diffraction of (111), (200), (220), (311) Ag planes, suggesting that the ultrathin Ag NWs are high quality and pure. ${ }^{\mathbf{1 4 3 4}}$

To further examine the effect of stirring speeds on the Ag NW synthesis, three different stirring speeds $(0,200$, and $400 \mathrm{rpm})$ for the same reaction time and temperature were carried out. Fig. 3a-f and S3a-c $\dagger$ shows the SEM images and length distribution of the $\mathrm{Ag}$ NWs obtained in $\mathrm{AgCl}$ and $\mathrm{NaBr}$ mixture under different stirring speed. The results show that the average length of $\mathrm{Ag}$ NWs at is $9 \mu \mathrm{m}$ at $0 \mathrm{rpm}, 11 \mu \mathrm{m}$ at $200 \mathrm{rpm}$, and 6 $\mu \mathrm{m}$ at $400 \mathrm{rpm}$, Fig. S3a-c $\dagger$ shows the average diameter is $44 \mathrm{~nm}$ at $0 \mathrm{rpm}, 30 \mathrm{~nm}$ at $200 \mathrm{rpm}$, and $39 \mathrm{~nm}$ at $400 \mathrm{rpm}$. Compared to the products at $0 \mathrm{rpm}$, the $\mathrm{Ag}$ NWs at $200 \mathrm{rpm}$ exhibit thinner diameter, longer length, and higher purity. Compared to the products at $200 \mathrm{rpm}$, the $\mathrm{Ag}$ NWs at $400 \mathrm{rpm}$ also present thick diameter, but shorter length and a small number of irregular particles were also observed, meaning that moderate magnetic stirring during the chemical reaction is a key regulator of the
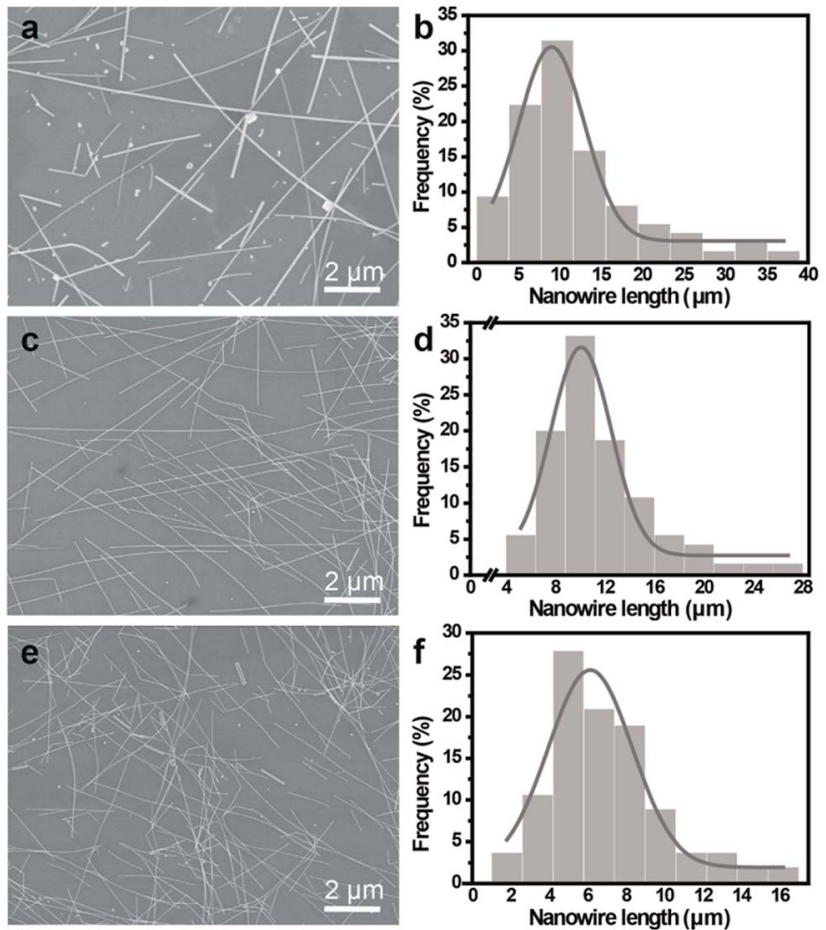

Fig. 3 SEM images and length distribution of Ag NWs prepared at different stirring speed: $(a, b)$ at 0 rpm the yield of Ag NWs is low, (c, d) at $200 \mathrm{rpm}$ results in high-yield formation of $\mathrm{Ag} \mathrm{NWs}$, and $(e, f)$ at 400 rpm Ag NWs with short length are formed. synthesis of high quality Ag NWs, with the additional merits of high yield, ultrathin diameter, and longer length. The yield of Ag NW product was measured by calculating the number densities of nanowires according the previous reported method. ${ }^{27}$ It was noted that the optimized process here provides access to produce large amounts of Ag NWs with a high yield up to $90 \%$ at $200 \mathrm{rpm}$. Therefore, high quality $\mathrm{Ag}$ NWs with an ultra-thin diameter in our polyol process could be realized through adjusting the mechanical agitation.

To evaluate the performance of Ag NW conductors, Ag NW films were fabricated by the spin-coating method on glass substrates. Fig. 4a shows the relationship between the transmittance and sheet resistances of the Ag NW films under ambient conditions. With ultra-thin $\mathrm{Ag}$ NWs prepared at $200 \mathrm{rpm}$, the sheet resistance and transmittance were $106.6 \Omega$ $\mathrm{sq}^{-1}$ and $95.1 \%$ at $550 \mathrm{~nm}$, respectively. A low sheet resistance of approximately $26 \Omega \mathrm{sq}^{-1}$ at $90.5 \%$ and $12.2 \Omega \mathrm{sq}^{-1}$ at $83.3 \%$ was obtained, which are superior to traditional ITO conductor and previously reported $\mathrm{Ag} \mathrm{NW}$ conductors $\left(20 \Omega \mathrm{sq}^{-1}\right.$ @ $80 \%$ at $550 \mathrm{~nm}^{7,33}$ and $19 \Omega \mathrm{sq}^{-1}$ (a $80 \%$ at $550 \mathrm{~nm}$ (ref. 11)). It is well known that the transmittance depends on the total area covered with Ag NWs on the substrate, hence, the transmittance loss of visible light owing to $\mathrm{Ag}$ NWs is approximately equal to the total area covered with the Ag NWs and the density dependence of the optical transmittance of an Ag NW film can be given by the following equation. ${ }^{33}$

$$
\% T=100 \times\left(1-A_{\mathrm{c}}\right)
$$
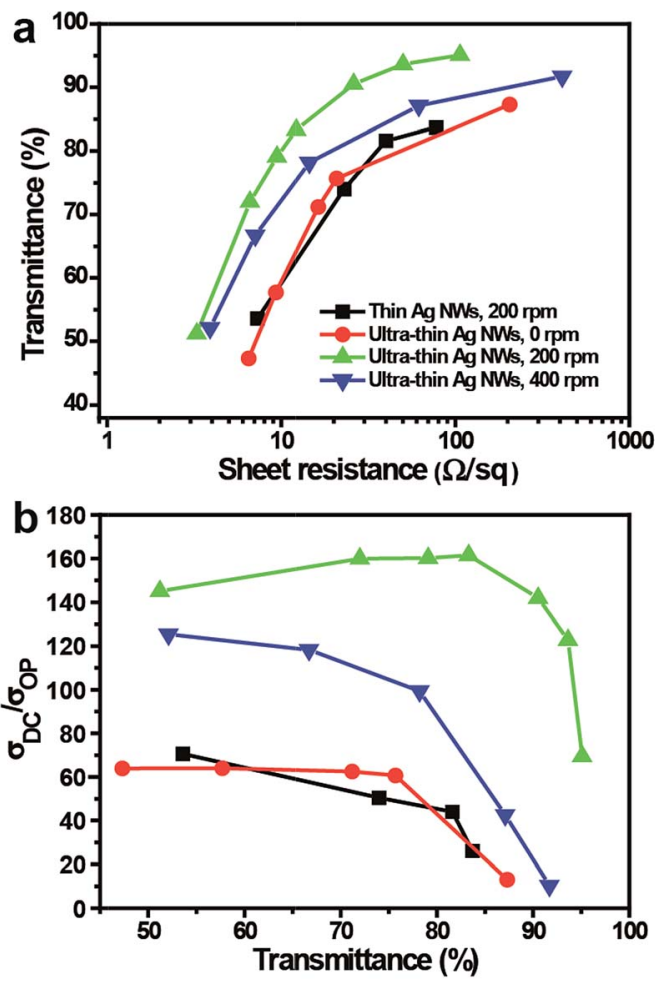

Fig. 4 (a) Measured $R_{\mathrm{s}}-T$ performance of transparent Ag NW conductors and (b) comparison of their corresponding $\sigma_{\mathrm{DC}} / \sigma_{\mathrm{OP}}$. 


$$
A_{\mathrm{c}}=L d
$$

where $A_{\mathrm{c}}$ is the total area covered with $\mathrm{Ag}$ NWs, $d$ is the diameter of an $\mathrm{Ag} \mathrm{NW}, N$ is number of wires per unit area. Generally, the transmittance and sheet resistance for thin conduct films are related by the eqn $(3)^{35,36}$

$$
\% T(\lambda)=\left(1+\frac{Z_{0}}{2 R_{\mathrm{s}}} \frac{\sigma_{\mathrm{OP}}}{\sigma_{\mathrm{DC}}}\right)^{-2}
$$

where $Z_{0}$ is the impedance of free space $(377 \Omega), \sigma_{\mathrm{OP}}$ is the optical conductivity and $\sigma_{\mathrm{DC}}$ is the DC conductivity of the films. The ratio of the DC conductivity to the optical conductivity, $\sigma_{\mathrm{DC}} /$ $\sigma_{\mathrm{OP}}$, has been used to evaluate the performance of transparent conductors. In Fig. $4 \mathrm{~b}$, the highest value of $\sigma_{\mathrm{DC}} / \sigma_{\mathrm{OP}}$ is $\sim 162$ for the ultrathin $\mathrm{Ag} \mathrm{NW}$ film $\left(R_{\mathrm{s}}=12.2 \Omega \mathrm{sq}^{-1}\right.$ and $\left.T=83.3 \%\right)$, which is much higher than the reported value $(\sim 70),{ }^{33}$ suggesting that ultrathin diameter and high aspect ratio are critical factors for high performance $\mathrm{Ag}$ NW conductors. Moreover, we have prepared flexible Ag NWs films based on NOA 63 substrate due to the ultra-smooth surface of substrate. ${ }^{38}$ The Ag NWs embedded NOA 63 films show superior mechanical flexibility, the sheet resistance change ratio $\left(R_{\mathrm{s}}-R_{\mathrm{s} 0}\right) / R_{\mathrm{s} 0}$ was almost unchanged during the bending test, as shown in Fig. S4, $\dagger$ indicating the $\mathrm{Ag}$ NW embedded NOA 63 films have potential applications in flexible electronics.

Haze is crucial in display applications where scattering will reduce the sharpness of an image, the haze factor of a transparent conducting films extremely dependent on nanowire diameter, with lower diameter nanowires featuring less haze. ${ }^{\mathbf{1 7}}$ We tested the haze of different diameter Ag NW films and Fig. 5 shows several haze values at different total transmittance values. The haze value decreased almost linearly with the increase of total transmittance for different diameter $\mathrm{Ag}$ NW films. For ultrathin $\mathrm{Ag} \mathrm{NW}$ films, the total transmittance is $91.6 \%$, and the haze factor is about $2 \%$, at total transmittance of $95.7 \%$, the haze factor is only $0.96 \%$. With the decrease of the diameter of the Ag NWs, the haze value increased at the same total transmittance. Compare to large diameter $\mathrm{Ag}$ NWs, the ultrathin Ag NW based films show lower haze and our result for

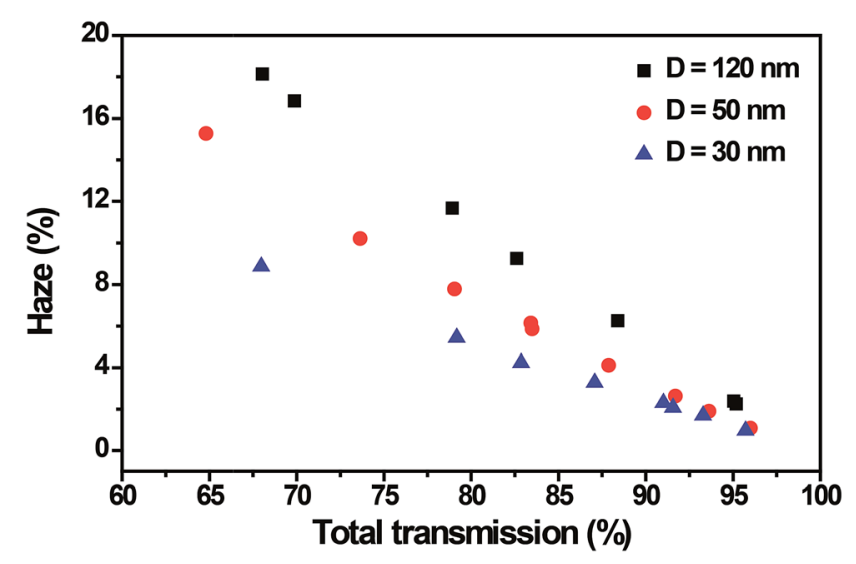

Fig. 5 The relationship between the haze and total transmission for different diameter Ag NW films. The conductors with ultrathin Ag NWs showed low haze of $2 \%$ and $91.6 \% T$ at wavelength of $550 \mathrm{~nm}$. ultrathin Ag NW films also showed significantly lower haze than any other Ag NW transparent conductor reported in the literature.

In summary, the effect of initial nucleation source and the stirring speeds for Ag NW synthesis through polyol method was studied. Compared to traditional polyol methods, reducing silver nitrate by using $\mathrm{AgCl}$ and $\mathrm{NaBr}$ as nucleation of silver seed results in rapid synthesis of ultrathin Ag NWs. Meanwhile, by adjusting the speed of the magnetic stirring, the yield of ultrathin Ag NW increased significantly, with a high value of $90 \%$. The produced Ag NWs exhibit an average diameter of $30 \mathrm{~nm}$ and an average length of $11 \mu \mathrm{m}$. By using the above nanowires, high performance Ag NW films with low sheet resistance and ultrahigh transmittance were achieved. The obtained $\mathrm{Ag} \mathrm{NW}$ conductor exhibits a sheet resistance of $26 \Omega \mathrm{sq}^{-1}$ at $90.5 \%$ and of $12.2 \Omega \mathrm{sq}^{-1}$ at $83.3 \%$. A value of $\sigma_{\mathrm{DC}} / \sigma_{\mathrm{OP}}=162$ is large enough for Ag NW conductors. Moreover, ultrathin Ag NW conductor exhibits a low haze of $2 \%$ and a low sheet resistance of $30 \Omega \mathrm{sq}^{-1}$ at a high optical transmittance of $91.6 \%$. The haze factor is $0.96 \%$ at a transmittance of $95.7 \%$. Such high-yield and rapid synthesis of ultrathin Ag NWs through the modified polyol method show great potential application in flexible electronics of the very near future.

\section{Acknowledgements}

This work was supported by the National Key Basic Research Program of China (973 Program, 2015CB932200), the National Natural Science Funds for Excellent Young Scholars (61622402), the National Natural Science Foundation of China (61376088, 51302134, 51528201), Natural Science Foundation of Jiangsu Higher Education Institutions (13KJB510012), the Natural Science Foundation of Jiangsu Province (BK20130934), and the Six Talent Plan (2015XCL015).

\section{Notes and references}

1 P. C. Hsu, S. Wang, H. Wu, V. K. Narasimhan, D. Kong, H. R. Lee and Y. Cui, Nat. Commun., 2013, 4, 2522.

2 J. Liang, L. Li, K. Tong, Z. Ren, W. Hu, X. Niu, Y. Chen and Q. Pei, ACS Nano, 2014, 8, 1590.

3 H. Guo, N. Lin, Y. Chen, Z. Wang, Q. Xie, T. Zheng, N. Gao, S. Li, J. Kang, D. Cai and D. L. Peng, Sci. Rep., 2013, 8, 421.

4 J. Jiu, T. Araki, J. Wang, M. Nogi, T. Sugahara, S. Nagao, H. Koga, K. Suganuma, E. Nakazawa, M. Hara, H. Uchida and K. Shinozaki, J. Mater. Chem. A, 2014, 2, 6326.

5 H. Kang, S. Jung, S. Jeong, G. Kim and K. Lee, Nat. Commun., 2015, 6, 6503.

6 M. S. Lee, K. Lee, S. Y. Kim, H. Lee, J. Park, K. H. Choi, H. K. Kim, D. G. Kim, D. Y. Lee, S. W. Nam and J. U. Park, Nano Lett., 2013, 13, 2814.

7 L. Hu, H. S. Kim, J. Y. Lee, P. Peumans and Y. Cui, ACS Nano, 2010, 4, 2955.

8 H. G. Lm, S. H. Jung, J. Jin, D. Lee, J. Lee, D. Lee, J. Y. Lee, I. D. Kim and B. S. Bae, ACS Nano, 2014, 8, 10973.

9 J. Song, J. Li, J. Xu and H. Zeng, Nano Lett., 2014, 14, 6298. 
10 J. Y. Lee, S. T. Connor, Y. Cui and P. Peumans, Nano Lett., 2008, 8, 689.

11 T. Araki, J. Jiu, M. Nogi, H. Koga, S. Nagao, T. Sugahara and K. Suganuma, Nano Res., 2014, 7, 236.

12 T. Kim, A. Canlier, G. H. Kim, J. Choi, M. Park and S. M. Han, ACS Appl. Mater. Interfaces, 2013, 5, 788.

13 C. Preston, Y. Xu, X. Han, J. N. Munday and L. Hu, Nano Res., 2013, 6, 461.

14 J. Y. Lin, Y. L. Hsueh and J. J. Huang, J. Solid State Chem., 2014, 214, 2.

15 J. Y. Lin, Y. L. Hsueh, J. J. Huang and J. R. Wu, Thin Solid Films, 2015, 584, 243.

16 R. Yang, C. Sui, J. Gong and L. Qu, Mater. Lett., 2007, 61, 900.

17 C. J. Murphy and N. R. Jana, Adv. Mater., 2002, 14, 80.

18 J. Lee, P. Lee, H. Lee, D. Lee, S. S. Lee and S. H. Ko, Nanoscale, 2012, 4, 6408.

19 P. Lee, J. Lee, H. Lee, J. Yeo, S. Hong, K. H. Nam, D. Lee, S. S. Lee and S. H. Ko, Adv. Mater., 2012, 24, 3326.

20 T. Tokuno, M. Nogi, J. Jiu and K. Suganuma, Nanoscale Res. Lett., 2012, 7, 281.

21 S. De, T. M. Higgins, P. E. Lyons, E. M. Doherty, P. N. Nirmalraj, W. J. Blau, J. J. Boland and J. N. Coleman, ACS Nano, 2009, 3, 1767.

22 J. Jiu, K. Murai, D. Kim, K. Kim and K. Suganuma, Mater. Chem. Phys., 2009, 114, 333.

23 W. Gaynor, G. F. Burkhard, M. D. McGehee and P. Peumans, Adv. Mater., 2011, 23, 2905.
24 J. Jiu, M. Nogi, T. Sugahara, T. Tokuno, T. Araki, N. Komoda, K. Suganuma, H. Uchidab and K. Shinozaki, J. Mater. Chem., 2012, 22, 23561.

25 E. J. Lee, M. H. Chang, Y. S. Kim and J. Y. Kim, APL Mater., 2013, 1, 042118.

26 S. M. Bergin, Y. H. Chen, A. R. Rathmell, P. Charbonneau, Z. Y. Li and B. J. Wiley, Nanoscale, 2012, 4, 1996.

27 X. Tang, M. Tsuji, P. Jiang, M. Nishio, S. M. Jang and S. H. Yoon, Colloids Surf., A, 2009, 338, 33.

28 Q. Zhang, Y. Hu, S. Guo, J. Goebl and Y. Yin, Nano Lett., 2010, 10, 5037.

29 Y. J. Song, M. Wang, X. Y. Zhang, J. Y. Wu and T. Zhang, Nanoscale Res. Lett., 2014, 9, 17.

30 J. P. Kottmann, O. J. F. Martin, D. R. Smith and S. Schultz, Phys. Rev. B: Condens. Matter Mater. Phys., 2001, 64, 235402. 31 Y. Sun and Y. Xia, Analyst, 2003, 128, 686.

32 J. J. Mock, S. J. Oldenburg, D. R. Smith, D. A. Schultz and S. Schultz, Nano Lett., 2002, 2, 465.

33 S. E. Park, S. Kim, D. Y. Lee, E. Kim and J. Hwang, J. Mater. Chem. A, 2013, 1, 14286.

34 A. G. Acosta, A. M. Ramírez, E. J. L. Naranjo, L. M. Apatiga, R. H. Basurto and E. M. R. Muñoz, Mater. Lett., 2015, 138, 167.

35 M. G. Dressel and G. Gruner, Electrodynamics of Solids: Optical properties of Electrons in Matter, Cambridge University Press, Cambridge, 2002.

36 W. He and C. Ye, J. Mater. Sci. Technol., 2015, 31, 581.

37 K. Zhang, Y. Du and S. Chen, Org. Electron., 2015, 26, 380. 38 Y. H. Duan, Y. Duan, X. Wang, D. Yang, Y. Q. Yang, P. Chen, F. B. Sun, K. W. Xue and Y. Zhao, Appl. Surf. Sci., 2015, 351, 445. 